\title{
Blood eosinophils: a biomarker of COPD exacerbation reduction with inhaled corticosteroids
}

This article was published in the following Dove Press journal: International Journal of COPD

\author{
Salman H Siddiqui ${ }^{1,2}$ \\ Ian D Pavord ${ }^{3,4}$ \\ Neil C Barnes ${ }^{5,6}$ \\ Alessandro Guasconi ${ }^{7}$ \\ Sally Lettis ${ }^{8}$ \\ Steven Pascoe 9 \\ Stefano Petruzzelli ${ }^{7}$ \\ 'University of Leicester, Leicester, \\ UK; ${ }^{2}$ National Institute for Health \\ Research Leicester Biomedical \\ Research Centre, Leicester, UK; \\ ${ }^{3}$ Respiratory Theme, National \\ Institute for Health Research Oxford \\ Biomedical Research Centre, Oxford \\ University Hospitals NHS Foundation \\ Trust, University of Oxford, Oxford, \\ UK; ${ }^{4}$ Respiratory Medicine Unit, \\ Nuffield Department of Medicine, \\ University of Oxford, Oxford, UK; \\ ${ }^{5} \mathrm{Glaxo}$ Smith Kline, Brentford, \\ London, UK; ${ }^{6}$ Barts and The London \\ School of Medicine and Dentistry, \\ London, UK; ${ }^{7}$ Chiesi Farmaceutici SpA, \\ Parma, Italy; ${ }^{8} \mathrm{GSK}$, Uxbridge, London, \\ UK; ${ }^{9} \mathrm{GSK}$, King of Prussia, PA, USA
}

Correspondence: Salman H Siddiqui National Institute for Health Research Leicester Biomedical Research Centre, Groby Road, Leicester LE3 9QP, UK Tel +44 II6 2502873

Email ss338@le.ac.uk
Background: Growing evidence suggests that blood eosinophil count is associated with patient responsiveness to inhaled corticosteroids (ICS). We performed post hoc predictive modeling on data from the FORWARD study and two replicate studies by Dransfield, to evaluate the relationships between baseline eosinophil count and the effect of ICS on exacerbations and lung function in patients with COPD.

Methods: The studies assessed ICS/long-acting $\beta_{2}$ agonist (LABA) combinations vs LABA alone. Using data from each study, we modeled COPD exacerbation rates, predose $\mathrm{FEV}_{1}$, and St George's Respiratory Questionnaire score ([FORWARD only]) over a continuous range of eosinophils (0-1,000 eosinophils/ $\mu \mathrm{L}$ in FORWARD, 0-993 eosinophils/ $\mu \mathrm{L}$ in Dransfield).

Results: In all studies, ICS/LABA reduced exacerbations versus LABA alone across all eosinophil levels, with progressively greater reductions at increasing baseline blood eosinophil counts. In FORWARD, annual exacerbation rates ranged from 0.78 to 0.83 per year between 0 and 1,000 eosinophils/ $\mu \mathrm{L}$ in the ICS/LABA arm, and from 0.81 to 1.54 per year in the LABAonly arm. In the Dransfield studies, exacerbation rates ranged from 0.54 to 1.02 per year in the ICS/LABA arm between 0 and 993 eosinophils/ $\mu \mathrm{L}$, and from 0.56 to 1.75 per year in the LABA-only arm. Change in $\mathrm{FEV}_{1}$ was not associated with eosinophil count in ICS-treated patients in FORWARD, whereas an increased treatment benefit in terms of FEV, was observed at higher eosinophil levels in the Dransfield studies. ICS/LABA led to greater improvements in St George's Respiratory Questionnaire total scores compared to LABA alone in patients in FORWARD with $\geq 67$ eosinophils/ $\mu \mathrm{L}$.

Conclusion: Higher blood eosinophil count in patients with COPD is associated with an increased beneficial effect from ICS in terms of exacerbation reduction. Further prospective data are required to assess the role of blood eosinophils as a biomarker for therapeutic recommendations.

Keywords: lung function, $\mathrm{FEV}_{1}$, COPD, ICS

\section{Introduction}

There has been considerable interest in the potential role of blood eosinophil count as a predictor of response to inhaled corticosteroids (ICS) in COPD, particularly with regard to prevention of exacerbations. ${ }^{1,2}$

Several studies investigating the predictive value of blood eosinophil count have been published. Pavord et al reviewed the association between blood eosinophils and exacerbation-rate reduction with ICS in the TRISTAN and INSPIRE studies. ${ }^{3}$ The TRISTAN study was a 1-year comparison of placebo, salmeterol, fluticasone propionate (FP), and combination $\mathrm{FP} /$ salmeterol in patients with moderate-to-severe COPD. The INSPIRE study was a 2-year comparison of tiotropium bromide (TIO) 
and $\mathrm{FP} /$ salmeterol in patients with severe or very severe COPD and history of exacerbations. ${ }^{3}$ A further analysis of the relationship between blood eosinophils and ICS response in the ISOLDE study was conducted by Barnes et $\mathrm{al} ;{ }^{4}$ the primary interest of this study was the relative effects of FP and placebo on the rate of decline of $\mathrm{FEV}_{1}$ over a 3-year period. ${ }^{5}$ Watz et al reported the results of a post hoc analysis on the association between blood eosinophils and exacerbation rate with ICS withdrawal in the WISDOM study, a double-blind randomized trial that incorporated a 9-month period when ICS were withdrawn or continued. ${ }^{6}$ Overall, six previous studies have consistently demonstrated that blood eosinophil count is associated with response to ICS. $^{1-4,7,8}$ However, to date, the published analyses have been mainly post hoc and retrospective in nature and have reported findings from trials of differing design that have used different statistical techniques to evaluate the impact of blood eosinophil count.

Three large, randomized controlled clinical trials (the FORWARD study [NCT00929851] and two studies by Dransfield [NCT01009463/NCT01017952]) $)^{9,10}$ that measured baseline blood eosinophils and shared similar study designs were completed between October 2011 and July 2012. The trials evaluated ICS/long-acting $\beta_{2}$ agonist (LABA) combination therapy and LABA alone over a 1-year period in patients with moderate-to-severe COPD. ${ }^{9,10}$ The FORWARD study compared a combination of beclomethasone dipropionate (BDP) and formoterol (FOR) with FOR alone, ${ }^{10}$ and the replicate Dransfield studies compared a combination of fluticasone furoate (FF) and vilanterol (VI) with VI alone. ${ }^{9}$

Although the FORWARD study and the Dransfield studies were of a similar design, two post hoc analyses of these studies used different approaches to categorize patients by baseline blood eosinophil count. ${ }^{7,8}$ Siddiqui et al analyzed the results of the FORWARD study according to quartiles of absolute eosinophil count ( 0 to $<110.4$ cells $/ \mu \mathrm{L}, 110.4$ to $<181.6$ cells $/ \mu \mathrm{L}, 181.6$ to $<279.8$ cells $/ \mu \mathrm{L}$, and $\geq 279.8$ cells $/ \mu \mathrm{L})$, quartiles of percentage of blood eosinophils ( $0 \%$ to $<1 \%, 1 \%$ to $<2 \%, 2 \%$ to $<3 \%$, and $>3 \%$ ), and an exploratory model of exacerbation risk as a function of absolute eosinophil count as a continuous distribution. ${ }^{8}$ In contrast, Pascoe et al analyzed the results of the Dransfield studies according to the percentage of eosinophils ( $<2 \%, 2 \%$ to $<4 \%, 4 \%$ to $<6 \%$, and $\geq 6 \%$ ) and absolute eosinophil count $(<150$ cells $/ \mu \mathrm{L}$ and $\geq 150$ cells $/ \mu \mathrm{L}){ }^{7}$

In the current study, we applied a consistent statistical modeling approach to data from the FORWARD and Dransfield studies to assess the effect of blood eosinophil count as a continuous variable on COPD outcomes in ICS- and non-ICS-treated patients. We also examined the reproducibility of the predictive model developed by Siddiqui et al using the Dransfield studies as a method of external model replication.

\section{Methods \\ Study objectives}

We aimed to analyze the results of the FORWARD and replicate Dransfield studies to 1 ) model baseline blood eosinophils as a continuous trait in these study populations, and evaluate the associated exacerbation risk and impact on lung function (assessed by $\mathrm{FEV}_{1}$ ) and quality of life (assessed by St George's Respiratory Questionnaire [SGRQ] total score) in the presence and absence of ICS and 2) determine the reproducibility of the prediction model developed by Siddiqui et al using the Dransfield studies as a method of external model replication. For our analysis, we used statistical techniques and models that were as similar as possible to one another, given the study design differences. This post hoc analysis was conducted as a collaboration between academic partners based in Leicester and Oxford, and the pharmaceutical companies GSK and Chiesi.

\section{Studies included in the analysis}

The studies examined in this analysis shared similar designs, comparing treatment with LABA alone with an ICS/LABA combination over a 48-52-week period. The FORWARD study compared FOR $12 \mu \mathrm{g}$ one inhalation, twice daily, with BDP/FOR 100/6 $\mu \mathrm{g}$ two inhalations, twice daily. ${ }^{10}$ The replicate Dransfield studies compared VI $25 \mu \mathrm{g}$ with FF/VI 50-200/25 $\mu \mathrm{g}$ once daily. ${ }^{9}$ The FORWARD and Dransfield studies were multinational studies that recruited patients with a history of exacerbations in the previous year, measured the annual rate of moderate-to-severe exacerbations as the primary outcome (those requiring a course of antibiotics/ corticosteroids and/or hospital admission), and measured $\mathrm{FEV}_{1}$. Only the FORWARD study measured SGRQ. ${ }^{9,10}$ The use of TIO was permitted only in the FORWARD study, and only if it was being taken prior to study entry.

The coprimary endpoints of the FORWARD study were moderate-to-severe exacerbation rate over 48 weeks and predose morning $\mathrm{FEV}_{1}$ at 12 weeks. The primary endpoint for the Dransfield studies was the annual rate of moderate-tosevere exacerbations. All three studies followed the European Medicines Agency/Committee for Medicinal Products for Human Use guideline for exacerbation definitions. The original trials utilized for this post hoc analysis had ethical 
and institutional review board approval, and all patients provided written informed consent. ${ }^{9,10}$

\section{Patients}

Patients included in the current analysis had moderate or severe COPD, with at least one exacerbation in the previous year. The Dransfield studies included patients with moderate or severe COPD (post-bronchodilator $\mathrm{FEV}_{1} /$ $\mathrm{FVC}$ ratio $<0.7$ and $\mathrm{FEV}_{1} \leq 70 \%$ predicted), whereas the FORWARD study included only patients with severe COPD (post-bronchodilator $\mathrm{FEV}_{1} / \mathrm{FVC}<0.7$ and $\mathrm{FEV}_{1} 30-<50 \%$ predicted).

\section{Statistical modeling}

Efficacy variables were analyzed for patients with available baseline eosinophil data in the intent-to-treat population (FORWARD study, $n=1,184$; Dransfield studies, $n=3,157$ ). The number of moderate and severe COPD exacerbations was analyzed using a negative binomial model with the number of exacerbations over the 48/52 weeks as the outcome. Fixed effects were treatment, country/region, smoking history, severity of airflow limitation (post-bronchodilator $\mathrm{FEV}_{1}$ at screening), and prior exacerbation frequency (one exacerbation, more than one exacerbation). The cubic root of baseline blood eosinophil count was used as a covariate, and log-time within the study as an offset variable. The interaction between treatment and cubic root of baseline blood eosinophil count was also included in the model. A cubic root transformation of the baseline eosinophil count was used to allow for the skewed distribution of eosinophil counts.

A linear mixed model for repeated measures for change from baseline in predose morning $\mathrm{FEV}_{1}$ at 48/52 weeks included treatment, visit, country/region, and smoking history as fixed effects, and baseline $\mathrm{FEV}_{1}$ value and cubic root of baseline blood eosinophil count as covariates. The interactions of treatment by visit, treatment by cubic root of baseline blood eosinophil count, visit by cubic root of baseline blood eosinophil count, and treatment by visit by cubic root of baseline blood eosinophil count were also included. An unstructured covariance matrix was assumed.

An analysis of covariance model for the change in SGRQ total score from baseline to 48 weeks included treatment, country, smoking history, baseline SGRQ total score, and cubic root of baseline blood eosinophil count as covariates. The interaction between treatment and cubic root of baseline blood eosinophil count was also included.

As patients in the FORWARD study could continue treatment with TIO during the study, a covariate adjusting for the effect of TIO was included in all FORWARD databased models. Separate analyses were conducted for the Dransfield studies (which were analyzed together) and the FORWARD study. For the former, the covariate "study" was included in order to adjust for the different sources of data (NCT01009463 or NCT01017952). Predictive modeling on the exacerbation rate (per person per year) in the FORWARD study has previously been reported. ${ }^{8}$

All statistical analyses were performed using SAS version 9.2 (SAS Institute, Cary, NC, USA).

\section{Results}

\section{Patients}

Patient demographics were comparable across the FORWARD and both Dransfield studies (Table 1). Compared to the FORWARD study population, a slightly higher proportion of patients in the Dransfield studies had experienced more than two recent exacerbations.

\section{Modeling of exacerbation rate}

Both the FORWARD and Dransfield exacerbation models predicted similar exacerbation rates with ICS/LABA vs LABA alone across the range of baseline eosinophil counts studied (Figure 1). In the FORWARD study, modeled annual exacerbation rates ranged from 0.78 to 0.83 per year between 0 and 1000 eosinophils/ $\mu \mathrm{L}$ in the ICS/LABA arm, and from 0.81 to 1.54 exacerbations per year in the LABA-only arm (Figure 1A). In the Dransfield studies, these rates ranged from 0.54 to 1.02 per year between 0 and 993 eosinophils/ $\mu \mathrm{L}$ in in the ICS/LABA arm, and 0.56-1.75 per year in the LABAonly arm (Figure 1B). In both models, patients at all baseline eosinophil levels had fewer annual exacerbations with ICS/ LABA vs LABA alone. As in the FORWARD study modeling, analyses on the Dransfield studies found that a higher baseline blood eosinophil count was associated with an increasing exacerbation risk in the absence of ICS treatment, while for patients treated with an ICS-containing regimen, the risk of developing an exacerbation was not influenced by the baseline blood eosinophil count (Figure 1). Both models showed that as baseline eosinophil levels increased, so did the treatment difference between ICS/LABA and LABA alone in terms of reduction in exacerbations.

\section{Modeling of change in FEV ,}

In the FORWARD study, patients treated with BDP/FOR had improvements from baseline in $\mathrm{FEV}_{1}$, and no association between baseline eosinophils and change from baseline in trough $\mathrm{FEV}_{1}$ was observed. In patients treated with FOR 
Table I Baseline characteristics of the intent-to-treat populations in the FORWARD and Dransfield studies

\begin{tabular}{|c|c|c|c|c|c|c|}
\hline & \multicolumn{2}{|c|}{ FORWARD (NCT0092985I) } & \multicolumn{4}{|c|}{ Dransfield studies (NCT0I009463/NCTOI0I7952, combined) } \\
\hline & $\begin{array}{l}\text { BDP/FOR } \\
100 / 6 \mu \mathrm{g} \\
(\mathrm{n}=594)\end{array}$ & $\begin{array}{l}\text { FOR } \\
12 \mu g \\
(n=590)\end{array}$ & $\begin{array}{l}\text { FF/VI } \\
50 / 25 \mu g \\
(\mathbf{n}=799)\end{array}$ & $\begin{array}{l}\text { FF/VI } \\
I 00 / 25 \mu g \\
(\mathbf{n}=783)\end{array}$ & $\begin{array}{l}\text { FF/VI } \\
200 / 25 \mu g \\
(n=778)\end{array}$ & $\begin{array}{l}\text { VI } \\
25 \mu g \\
(n=797)\end{array}$ \\
\hline Mean age, years $\pm S D$ & $64.6 \pm 8.7$ & $64.0 \pm 8.6$ & $63.7 \pm 9.3$ & $63.9 \pm 9.1$ & $63.6 \pm 9.0$ & $63.6 \pm 9.4$ \\
\hline Females, n (\%) & $186(31.3)$ & $181(30.7)$ & $334(4 I .8)$ & $342(43.7)$ & $329(42.3)$ & $335(42.0)$ \\
\hline $\begin{array}{l}\text { Mean post-bronchodilator } \mathrm{FEV}_{1} \text {, } \\
\mathrm{L} \pm \mathrm{SD}\end{array}$ & $1.15 \pm 0.3$ & 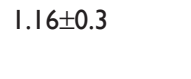 & $1.3 \pm 0.5$ & $1.3 \pm 0.5$ & $1.3 \pm 0.5$ & $1.3 \pm 0.5$ \\
\hline $\begin{array}{l}\text { Mean post-bronchodilator } \mathrm{FEV}_{1} \text {, } \\
\% \text { predicted } \pm \text { SD }\end{array}$ & $41.9 \pm 5.9$ & $41.6 \pm 6.0$ & $45.3 \pm 13.6$ & $46.0 \pm 13.4$ & $45.2 \pm 13.5$ & $45.2 \pm 13.1$ \\
\hline $\begin{array}{l}\text { Any moderate-to-severe } \\
\text { exacerbation, } n(\%) \\
0^{\text {a }} \\
1 \\
2 \\
>2\end{array}$ & $\begin{array}{l}0(0) \\
414(69.7) \\
131(22.1) \\
49(8.2)\end{array}$ & $\begin{array}{l}\text { I }(0.2) \\
415(70.3) \\
126(2 I .4) \\
48(8.1)\end{array}$ & $\begin{array}{l}0(0) \\
463(57.9) \\
219(27.4) \\
117(14.6)\end{array}$ & $\begin{array}{l}0(0) \\
487(62.2) \\
158(20.2) \\
138(17.6)\end{array}$ & $\begin{array}{l}2(0.3) \\
493(63.4) \\
182(23.4) \\
101(13.0)\end{array}$ & $\begin{array}{l}\text { I }(0.1) \\
471(59.1) \\
197(24.7) \\
128(16.1)\end{array}$ \\
\hline $\begin{array}{l}\text { Baseline absolute eosinophil count, } \\
\text { mean eosinophils/ } \mu \mathrm{L} \pm \mathrm{SD} \\
\text { Median eosinophils/ } \mu \mathrm{L}(\mathrm{Q} \text { I, Q3) }\end{array}$ & $\begin{array}{l}215 \pm 155 \\
180(112,273)\end{array}$ & $\begin{array}{l}219 \pm 161 \\
186(110,284)\end{array}$ & $\begin{array}{l}248 \pm 221 \\
200(120,310)\end{array}$ & $\begin{array}{l}252 \pm 261 \\
190(120,300)\end{array}$ & $\begin{array}{l}243 \pm 213 \\
190(110,310)\end{array}$ & $\begin{array}{l}232 \pm 197 \\
180(110,280)\end{array}$ \\
\hline 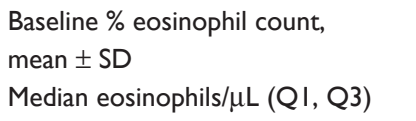 & $\begin{array}{l}2.8 \pm 1.9 \\
2.4(1.5,3.5)\end{array}$ & $\begin{array}{l}2.9 \pm 2.2 \\
2.4(1.5,3.9)\end{array}$ & $\begin{array}{l}3.3 \pm 2.7 \\
2.8(1.6,4.2)\end{array}$ & $\begin{array}{l}3.4 \pm 3.0 \\
2.7(1.6,4.3)\end{array}$ & $\begin{array}{l}3.3 \pm 2.7 \\
2.6(1.6,4.1)\end{array}$ & $\begin{array}{l}3.1 \pm 2.5 \\
2.5(I .5,3.9)\end{array}$ \\
\hline $\begin{array}{l}\text { Mean smoking exposure, } \\
\text { pack-years } \pm S D\end{array}$ & $43.1 \pm 23.5$ & $42.7 \pm 22.9$ & $46.2 \pm 26.6$ & $46.8 \pm 27.7$ & $46.5 \pm 29.7$ & $45.6 \pm 26.8$ \\
\hline
\end{tabular}

Note: aMajor protocol violators.

Abbreviations: BDP, beclomethasone dipropionate; FF, fluticasone furoate; FOR, formoterol; Q, quartile; VI, vilanterol.

alone, those with higher baseline eosinophil counts had greater improvements in $\mathrm{FEV}_{1}$ vs those with lower eosinophil counts; these improvements were lower than those observed in the BDP/FOR group (Figure 2A). The Dransfield study models showed that FF/VI improved airflow limitation vs VI alone for patients at almost all baseline blood eosinophil levels studied ( $\geq 22$ eosinophils $/ \mu L$ ). Patients with higher baseline blood eosinophil counts had the greatest improvement in $\mathrm{FEV}_{1}$ when treated with FF/VI, and the greatest loss in $\mathrm{FEV}_{1}$ with VI alone (Figure 2B).

\section{Modeling of change in SGRQ}

In the FORWARD study, BDP/FOR led to greater improvements in SGRQ total score vs FOR alone in patients with $\geq 67$ blood eosinophils $/ \mu \mathrm{L}$ at baseline (Figure 3). Patients in the BDP/FOR arm with higher baseline eosinophil counts had greater improvements in SGRQ total score compared to those with lower eosinophil counts. In the FOR arm, increasing baseline eosinophil counts were associated with worsening SGRQ total scores, and patients with baseline eosinophil counts of 306 eosinophils $/ \mu \mathrm{L}$ and above experienced a deterioration in their health status with FOR alone.

\section{Discussion}

In analyses of data from the FORWARD and Dransfield studies, patients treated with ICS/LABA demonstrated similar COPD exacerbation rates and lung function across all baseline blood eosinophil levels, with better outcomes compared to LABA alone.

When a model identical to that previously applied to data from the FORWARD study was applied to data from the Dransfield studies, the observations for exacerbations were similar to those of the FORWARD modeling. As baseline blood eosinophil count increased, so did exacerbation rate in the absence of ICS treatment. When treated with ICS/LABA, patients had similar exacerbation rates regardless of baseline blood eosinophil level. Therefore, the relative reduction of exacerbation rate provided by ICS becomes progressively greater with increasing baseline blood eosinophil counts.

In line with the exacerbation rate modeling results,

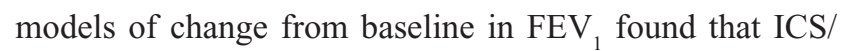
LABA improved lung function when compared to LABA alone across all baseline eosinophil levels in the FORWARD model, and across the majority of baseline eosinophil levels in the Dransfield study. In the FORWARD analysis, patients 
A

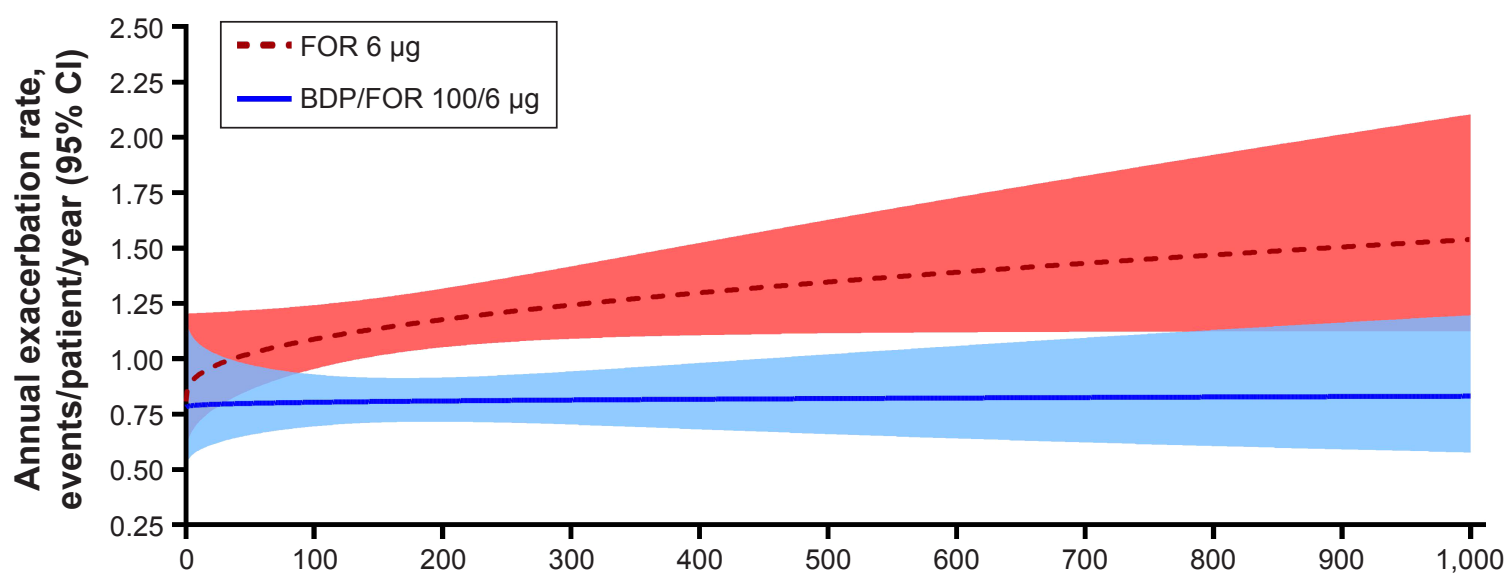

Absolute blood eosinophils at baseline (per $\mu \mathrm{L}$ )

B

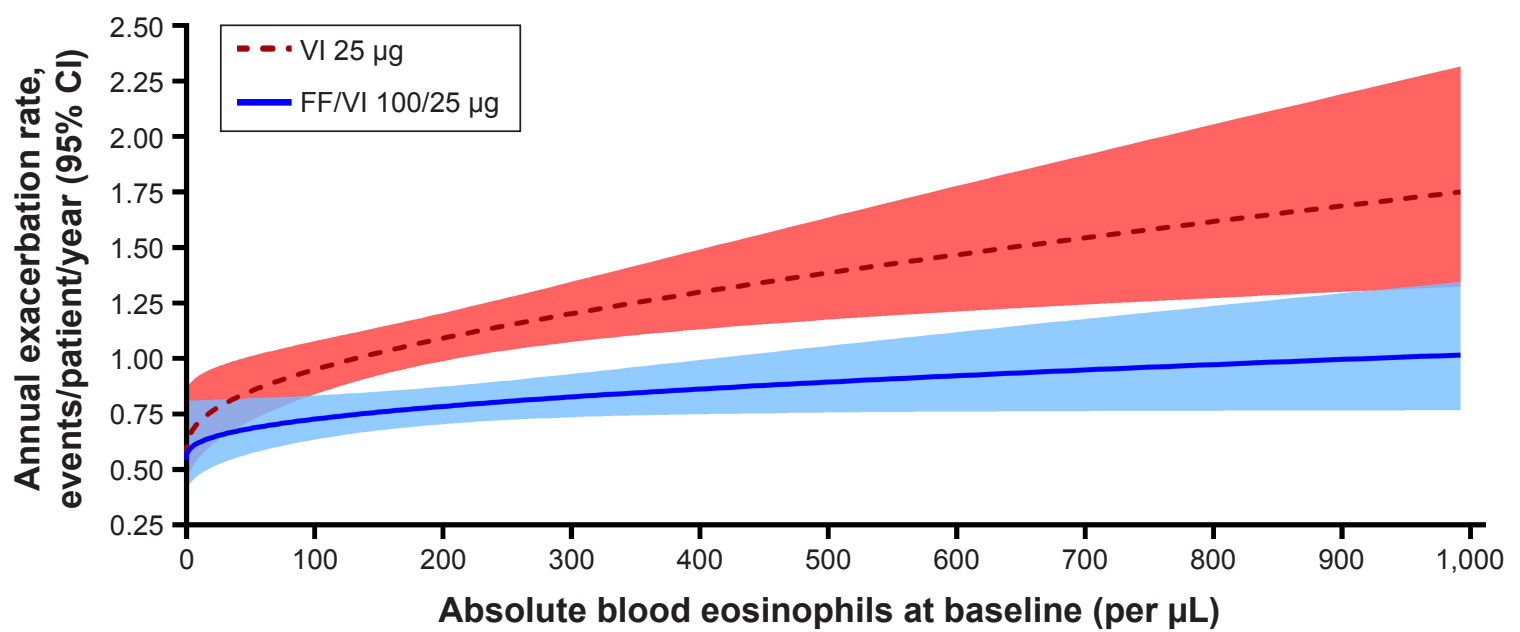

Figure I (A) Predicted exacerbation rates by baseline blood eosinophils for the FORWARD study; (B) predicted annual exacerbation rates by baseline blood eosinophils for the Dransfield studies.

Note: The shaded areas indicate the $95 \% \mathrm{Cl}$.

Abbreviations: BDP, beclomethasone dipropionate; FF, fluticasone furoate; FOR, formoterol; VI, vilanterol.

treated with ICS/LABA had improvements in $\mathrm{FEV}_{1}$ that were unaffected by baseline eosinophil level, whereas those treated with LABA alone only had improvements when at or above 231 eosinophils $/ \mu \mathrm{L}$. The Dransfield analyses found that, at higher baseline eosinophil counts, patients treated with ICS/LABA had the greatest improvements in FEV relative to patients treated with $\mathrm{LABA}$ alone.

In the analyses of SGRQ data from the FORWARD study, BDP/FOR was more effective than FOR alone at improving health status in patients with $\geq 67$ blood eosinophils $/ \mu \mathrm{L}$ at baseline. Patients in the BDP/FOR arm with higher baseline eosinophil counts had greater improvements in health status compared to those with lower eosinophil counts, whereas the treatment benefits of FOR alone reduced as eosinophil count increased. Patients with 306 eosinophils $/ \mu \mathrm{L}$ or above at baseline, roughly corresponding to the highest eosinophil quartile, who were treated with FOR alone, experienced a deterioration in their health status. At very low eosinophil counts, ICS/LABA appeared to negatively impact SGRQ total score compared to LABA alone; however, this pattern was likely a result of the limited number of SGRQ data points for patients with very low eosinophil counts.

Several investigative studies have assessed the potential role of eosinophils as a biomarker for ICS responsiveness, with the aim of identifying those patients who may not benefit from the use of ICS. ${ }^{3,10}$ Previous studies have used arbitrary thresholds for blood eosinophil count without analyzing the complete range. A review of three studies, including INSPIRE and TRISTAN, evaluated the benefits of FP/salmeterol vs the long-acting muscarinic antagonist (LAMA), TIO, or placebo 
A
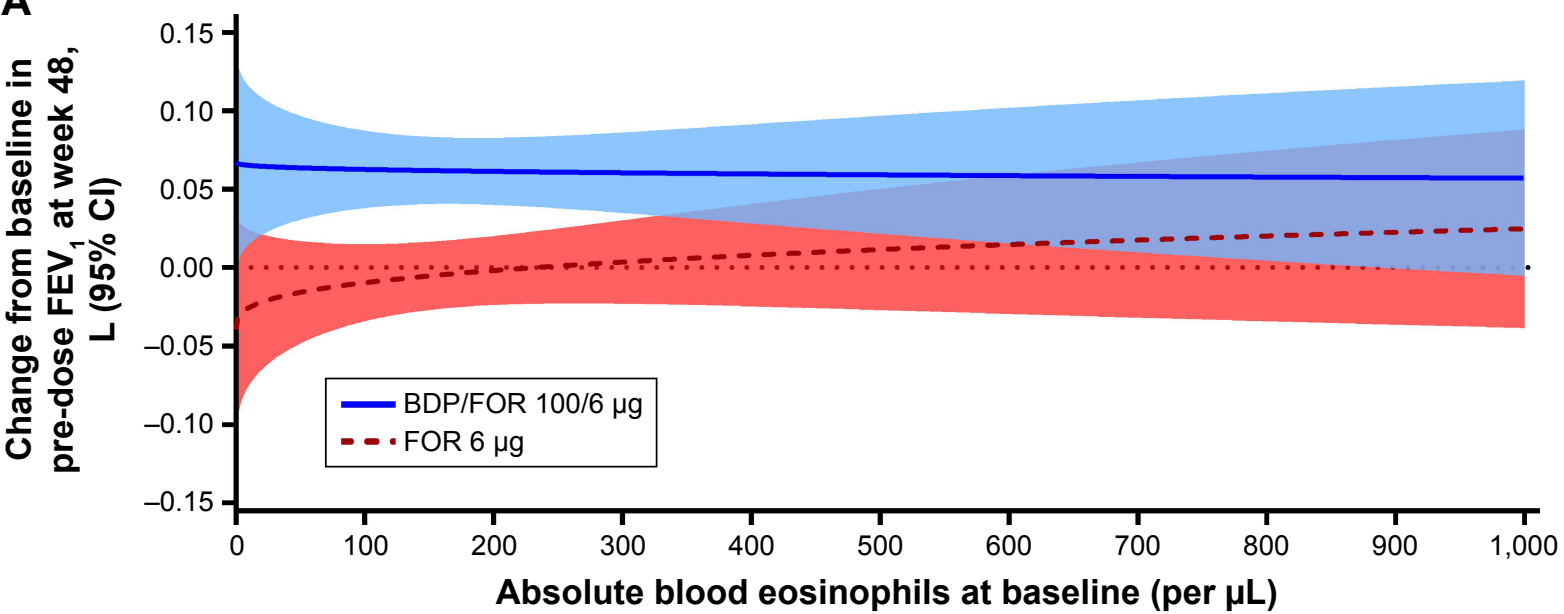

B

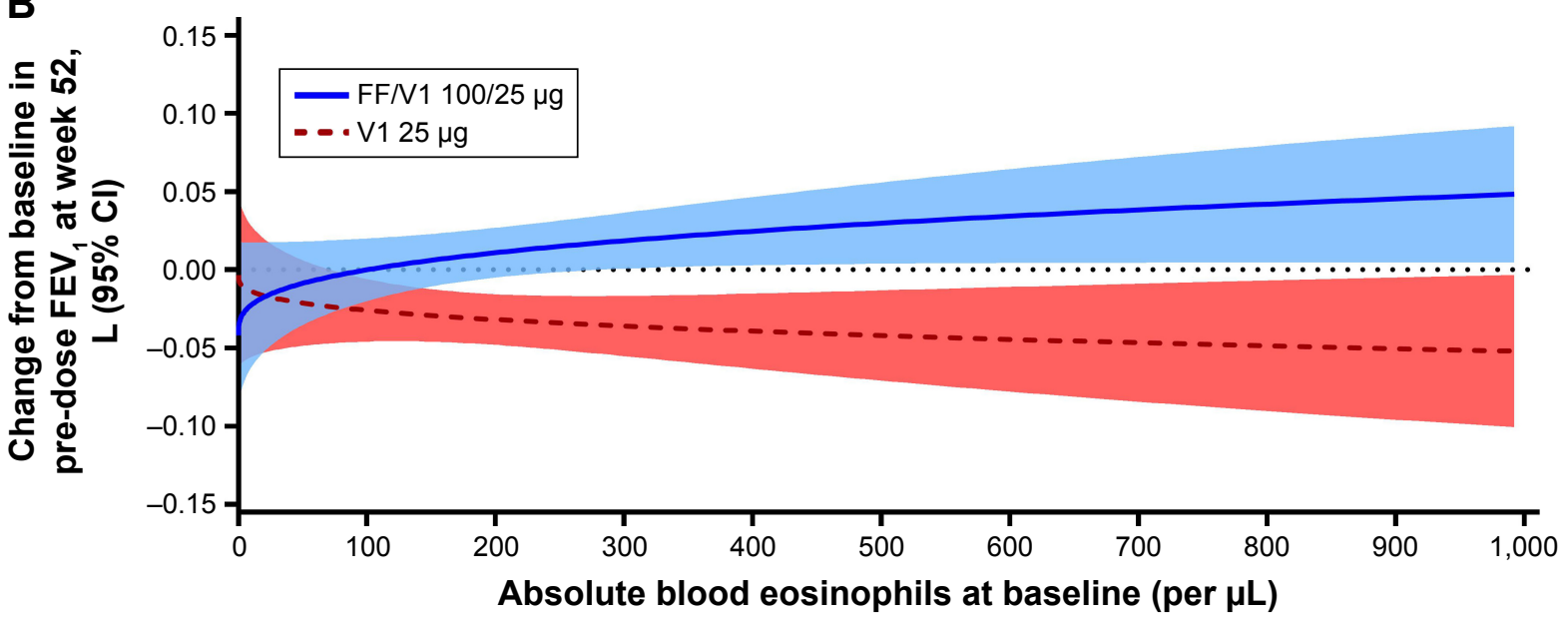

Figure 2 (A) Predicted change from baseline in trough FEV, by baseline blood eosinophils in the FORWARD study at week 48; (B) predicted change from baseline in trough FEV by baseline blood eosinophils in the Dransfield studies at week 52.

Note: The shaded areas indicate the $95 \% \mathrm{Cl}$.

Abbreviations: BDP, beclomethasone dipropionate; FF, fluticasone furoate; FOR, formoterol; VI, vilanterol.

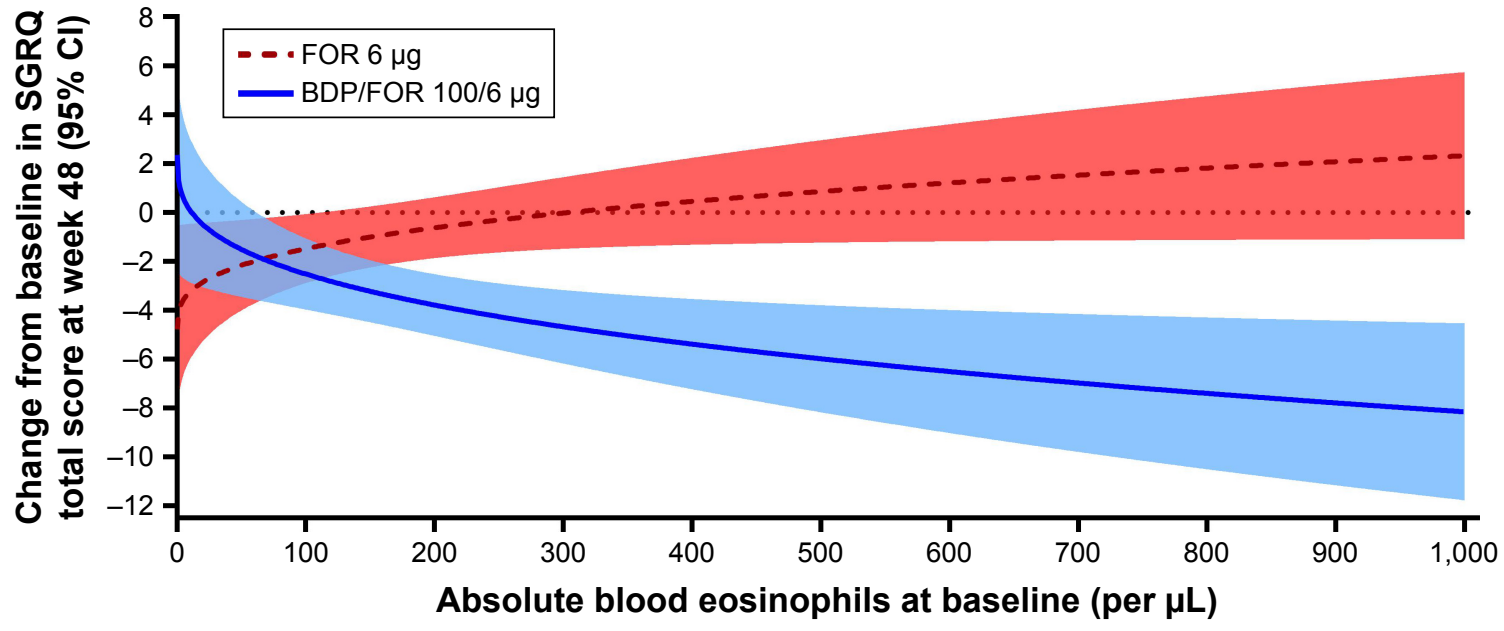

Figure 3 Predicted change from baseline in SGRQ total score at week 48 by baseline blood eosinophils in the FORWARD study. Note: The shaded areas indicate the $95 \% \mathrm{Cl}$.

Abbreviations: BDP, beclomethasone dipropionate; FOR, formoterol; SGRQ, St George's Respiratory Questionnaire. 
according to baseline eosinophil count. ${ }^{3} \mathrm{FP} /$ salmeterol was generally preferable to LAMA or placebo in reducing exacerbations in patients with $\geq 2 \%$ eosinophils, in agreement with our exacerbation rate modeling. However, contrary to the results of the current analyses, the review found no relationship between eosinophils and change from baseline in $\mathrm{FEV}_{1}$ or SGRQ with ICS/LABA vs LAMA or placebo.

The recent FLAME trial investigated the effects of indacaterol/glycopyrronium (LAMA/LABA) vs FP/salmeterol (ICS/LABA) on COPD exacerbation rates, stratifying patients according to eosinophil level ( $<2 \%$ and $\geq 2 \%$ ). Indacaterol/ glycopyrronium was found to be superior to $\mathrm{FP} /$ salmeterol in both eosinophil strata. ${ }^{11}$ In keeping with our analyses, the between-treatment differences lessened with increasing blood eosinophils. However, as the two treatment arms employed different LABA treatments, the results are difficult to interpret. The observed differences between the results of the FLAME trial and the FORWARD/Dransfield trials are likely to be a result of variation in study designs (for example, the FLAME trial excluded patients with $>600$ eosinophils $/ \mu \mathrm{L}$ and included a TIO run-in period unlike the Dransfield trials), as well as the possibility that LAMAs may have modified eosinophilia in the FLAME trial.

There are a number of limitations to this post hoc analysis, not least the limited sizes of the populations modeled, particularly the low number of patients with very high or very low eosinophil counts at baseline. The FORWARD and Dransfield studies did not select patients based on eosinophil count. Therefore, although the range of baseline eosinophil levels observed is representative of the usual population of patients with COPD, the results of this study for patients with very high or very low eosinophil counts should be viewed with caution. Furthermore, in both exacerbation rate models, the interaction between baseline eosinophils and treatment was nonsignificant, and the effect of baseline eosinophils was nonsignificant in the FORWARD exacerbation model. This is most likely due to a lack of statistical power rather than a weak association between baseline blood eosinophil count and treatment. These outcomes were not unexpected, as the previously published FORWARD modeling demonstrated a nonsignificant interaction between baseline blood eosinophils and treatment. ${ }^{8}$ To overcome the limitations of these analyses, further investigations are needed. Additionally, comparisons of ICS/LABA or ICS/LAMA/LABA vs LAMA/LABA would be of clinical interest.

\section{Conclusion}

When taking into account the combined modeling analyses of the FORWARD and Dransfield study datasets, patients with COPD and higher baseline blood eosinophil counts derived the greatest benefit from ICS therapy in terms of improvement in exacerbations. The role of eosinophils in the improvement of airflow and health status with ICS therapy is not clear. Further investigations are required to clearly define the role of eosinophils in clinical practice.

\section{Data availability}

The manuscript reports results from an analysis of three studies: "FORWARD” NCT00929851 (Chiesi) and replicate Dransfield studies NCT01009463/NCT01017952 (GSK). For GSK clinical trials, within 6 months of publishing the results of the primary endpoints of the study, anonymized individual participant data plus the annotated case report form, protocol, reporting and analysis plan, data set specifications, raw dataset, analysis-ready dataset, and clinical study report are available for research proposals approved by an independent review committee. Proposals should be submitted to www. clinicalstudydatarequest.com. A data access agreement will be required. From January 1, 2019, Chiesi commits to sharing the anonymized, patient-level data, study-level data, the clinical protocol, and the full clinical study report of the FORWARD study (NCT00929851) with qualified scientific and medical researchers, conducting legitimate research. Chiesi provides access to clinical trial information consistently with the principle of safeguarding commercially confidential information and patient privacy. Fundamental conditions for providing the requested clinical trial data are that qualified researchers agree to sign a Data Sharing Agreement, to use the data only for noncommercial purposes and to seek publication of their research results. Other information on Chiesi's data sharing commitment, access and research request's approval process will be available from January 1, 2019, in the Clinical Trial Transparency section of this webpage page: http:// www.chiesi.com/en/research-and-development/.

\section{Acknowledgments}

The authors would like to thank Amy Newlands, statistician at GSK, who performed the analyses. Editorial support (in the form of writing assistance, assembling tables and figures, collating author comments, grammatical editing, and referencing) was provided by Catherine Elliott and David Mayes at Gardiner-Caldwell Communications (Macclesfield), and was funded by GSK. The abstract of this paper was presented at the European Respiratory Society International Congress 2016 as an oral presentation with interim findings. The abstract was published in the Eur Respir J. 2016;48(60):OA1763: http://erj.ersjournals.com/ content/48/suppl 60/OA1763. This analysis was sponsored 
by GlaxoSmithKline plc (NCT01009463/NCT01017952) and Chiesi Farmaceutici SpA (NCT00929851) and was supported by the Leicester National Institute for Health Research (NIHR) Biomedical Research Centre: Respiratory Theme. The sponsors participated in the conception and design of the study, analysis and interpretation of the data, drafting and critical revision of the report, and approved submission of the manuscript. All authors had access to the results of the analyses, reviewed and edited the manuscript, approved the final draft, and were involved in the decision to submit the manuscript for publication. The views expressed are those of the authors and not necessarily those of the NHS, the NIHR, or the Department of Health.

\section{Author contributions}

Conception or design of the analysis and data analysis or interpretation: SHS, IDP, NCB, SL, SPa, AG, and SPe. SHS accepts responsibility for the content of the manuscript, including the data and analysis. All authors contributed to data analysis, drafting or revising the article, gave final approval of the version to be published, and agree to be accountable for all aspects of the work.

\section{Disclosure}

SHS has received grants from the Chiesi Onulus scheme to study small airways and is on the international steering board for the Chiesi-sponsored ATLANTIS trial; in the past 5 years, SHS has received honoraria for attending advisory panels with Roche, AstraZeneca, Boehringer Ingelheim, and GSK and performed consultancy for Owlstone Nanotech and Mundipharma; SHS has also received honoraria for speaking at sponsored meetings from AstraZeneca, Boehringer Ingelheim, GSK, and Novartis. IDP has received honoraria in the past 5 years for speaking at sponsored meetings from AstraZeneca, Boehringer Ingelheim, Aerocrine, Almirall, Novartis, and GSK and a payment for organizing an educational event from AstraZeneca; he has received honoraria for attending advisory panels with Almirall, Genentech, Regeneron, AstraZeneca, Boehringer Ingelheim, GSK, Merck Sharp \& Dohme, Schering-Plough, Novartis, Dey Pharma, Napp Pharmaceuticals, and RespiVert; he has received sponsorship to attend international scientific meetings from Boehringer Ingelheim, GSK, AstraZeneca, and Napp Pharmaceuticals. NCB is employed by GSK and holds GSK stocks. SL is employed by GSK and holds GSK stocks. AG is employed by Chiesi. SPa is employed by GSK and holds GSK stocks. SPe is employed by Chiesi. The authors report no other conflicts of interest in this work.

\section{References}

1. Price D, Rigazio A, Postma D. Blood eosinophilia and the number of exacerbations in COPD patients [abstract]. Eur Respir J. 2014;44(Suppl 58): 4416.

2. Vedel-Krogh S, Nielsen SF, Lange P, Vestbo J, Nordestgaard BG. Blood eosinophils and exacerbations in chronic obstructive pulmonary disease. The Copenhagen General Population Study. Am J Respir Crit Care Med. 2016;193(9):965-974.

3. Pavord ID, Lettis S, Locantore N, et al. Blood eosinophils and inhaled corticosteroid/long-acting $\beta$-2 agonist efficacy in COPD. Thorax. 2016; 71(2):118-125.

4. Barnes NC, Sharma R, Lettis S, Calverley PM. Blood eosinophils as a marker of response to inhaled corticosteroids in COPD. Eur Respir J. 2016;47(5):1374-1382.

5. Burge PS, Calverley PM, Jones PW, Spencer S, Anderson JA, Maslen TK. Randomised, double blind, placebo controlled study of fluticasone propionate in patients with moderate to severe chronic obstructive pulmonary disease: the ISOLDE trial. BMJ. 2000;320(7245): 1297-1303.

6. Watz H, Tetzlaff K, Wouters EF, et al. Blood eosinophil count and exacerbations in severe chronic obstructive pulmonary disease after withdrawal of inhaled corticosteroids: a post-hoc analysis of the WISDOM trial. Lancet Respir Med. 2016;4(5):390-398.

7. Pascoe S, Locantore N, Dransfield MT, Barnes NC, Pavord ID. Blood eosinophil counts, exacerbations, and response to the addition of inhaled fluticasone furoate to vilanterol in patients with chronic obstructive pulmonary disease: a secondary analysis of data from two parallel randomised controlled trials. Lancet Respir Med. 2015;3(6):435-442.

8. Siddiqui SH, Guasconi A, Vestbo J, et al. Blood eosinophils: a biomarker of response to extrafine beclomethasone/formoterol in chronic obstructive pulmonary disease. Am J Respir Crit Care Med. 2015;192(4): 523-525.

9. Dransfield MT, Bourbeau J, Jones PW, et al. Once-daily inhaled fluticasone furoate and vilanterol versus vilanterol only for prevention of exacerbations of COPD: two replicate double-blind, parallel-group, randomised controlled trials. Lancet Respir Med. 2013;1(3):210-223.

10. Wedzicha JA, Singh D, Vestbo J, et al; FORWARD Investigators. Extrafine beclomethasone/formoterol in severe COPD patients with history of exacerbations. Respir Med. 2014;108(8):1153-1162.

11. Wedzicha JA, Banerji D, Chapman KR, et al; FLAME Investigators. Indacaterol-Glycopyrronium versus Salmeterol-Fluticasone for COPD. N Engl J Med. 2016;374(23):2222-2234.
International Journal of COPD

\section{Publish your work in this journal}

The International Journal of COPD is an international, peer-reviewed journal of therapeutics and pharmacology focusing on concise rapid reporting of clinical studies and reviews in COPD. Special focus is given to the pathophysiological processes underlying the disease, intervention programs, patient focused education, and self management protocols.

\section{Dovepress}

This journal is indexed on PubMed Central, MedLine and CAS. The manuscript management system is completely online and includes a very quick and fair peer-review system, which is all easy to use. Visit $\mathrm{http}: / / \mathrm{www}$.dovepress.com/testimonials.php to read real quotes from published authors. 